\title{
The depth-profiled carrier concentration and scattering mechanism in undoped GaN film grown on sapphire
}

\author{
Y. Huang, X. D. Chen, S. Fung, ${ }^{\text {a) }}$ C. D. Beling, C. C. Ling, Z. F. Wei, S. J. Xu, \\ and C. Y. Zhi \\ Department of Physics, The University of Hong Kong, Pokfulam Road, Hong Kong, \\ People's Republic of China
}

(Received 19 December 2003; accepted 28 April 2004)

\begin{abstract}
Temperature-dependent Hall (TDH) measurements and confocal micro-Raman spectroscopy have been used to study the free carrier spatial distribution and scattering mechanism in unintentionally doped GaN film grown on the sapphire substrate with the method of metalorganic chemical vapor deposition. Both the TDH data and the depth-profiled Raman spectra agreed with the existence of a nonuniform spatial distribution of free carriers in the GaN film with a highly conductive layer of $\sim 1 \mu \mathrm{m}$ thickness near the GaN sapphire boundary. With the consideration of this parallel conduction channel adjacent to GaN sapphire boundary, detailed analysis of the TDH mobility data suggests that a relatively high concentration of nitrogen vacancies exists and nitrogen vacancy scattering has an important influence on limiting the electron mobility in the bulk film of the present GaN sample. (C) 2004 American Institute of Physics. [DOI: 10.1063/1.1763235]
\end{abstract}

\section{INTRODUCTION}

$\mathrm{GaN}$ has attracted considerable research interest because of its potential applications including blue/ultraviolet light emitting devices, ultraviolet detectors, ${ }^{1,2}$ high-speed, hightemperature field-effect transistors, and high electron mobility transistors. ${ }^{3-5} \mathrm{GaN}$ is desirable for high-power, highspeed electronic applications due to its higher breakdown field and larger saturation velocity than GaAs, and also its excellent ballistic conduction under a high electric field $(>100 \mathrm{kV} / \mathrm{cm}) .{ }^{6}$ Understanding the electronic transport properties of GaN such as Hall carrier mobility and scattering mechanism is therefore of high importance. Unfortunately such studies are complicated by the poor structural quality of GaN films arising from lattice-mismatched heteroepitaxy (13.8\% for sapphire) and the existence of additional parallel conducting channels in the material, such as through impurity bands, interfacial regions, or grain boundaries.

High quality GaN epilayers have been grown on sapphire substrates by introducing a low-temperature grown AlN or a GaN buffer layer. ${ }^{7-9}$ This results in the transport properties of $\mathrm{GaN}$ epilayer being shunted by a dislocated interface region with highly conductive properties. A twolayer model, which involves a thin degenerate interfacial layer and the GaN bulk layer, was constructed by Look and Molnar to successfully explain the Hall data although this two-layer model has relied on some assumptions. ${ }^{10}$ Using different scanning probe microscopic techniques, Hsu et al. ${ }^{11}$ directly observed an interfacial region at the GaN/sapphire boundary having an electron concentration at least an order of magnitude higher than that of the less-conducting bulk film. Evidence for such a degenerate interfacial layer also comes from the depth-profiled temperature-dependent Hall experiments performed by Mavroidis et al. ${ }^{12}$ Excess oxygen

${ }^{a)}$ Electronic mail: sfung@hkucc.hku.hk atoms diffusing from the sapphire substrate and defective microstructure have been suggested to be responsible for the high electron concentration in the interfacial layer. ${ }^{13-16}$

Raman spectroscopy is not only a dominant method for studying phonons, but also provides a contactless and nondestructive method for determining carrier concentration and mobility. In Raman spectroscopic studies of $\mathrm{GaN}$ materials, strong couplings between the longitudinal optical (LO) phonon and the free carrier plasma were observed. ${ }^{17,18}$ The LO phonon-plasmon coupling has the effects of shifting the LO phonon peak to high-frequency side and broadening the line shape. ${ }^{17,18}$ With this principle, Ponce et al. ${ }^{19}$ demonstrated how Raman spectroscopy can be used to probe the spatial variation of carrier concentration in Si doped $\mathrm{GaN}$.

In the present work, depth-profiled confocal microRaman spectroscopy was used to study the depth distribution of the carrier density across the GaN film grown on the sapphire substrate. Temperature-dependent Hall (TDH) measurements conducted on the same sample were fitted by the two-layer model, in which the first layer is the bulk film and the second one is the degenerate conductive layer. Results from both techniques agree with the two-layer model and the carrier concentrations of the two layers (i.e., GaN bulk layer and interfacial layer) derived from the two techniques coincide well with each other. With considering the highly conductive interfacial layer, analysis of the temperaturedependent mobility in the bulk layer incorporating the influence of various scattering processes reveals an important contribution coming from nitrogen vacancy scattering.

\section{EXPERIMENT}

The samples used in the present work were unintentionally doped GaN film grown on c-plane sapphire using ammonia $\left(\mathrm{NH}_{3}\right)$ and trimethylgallium (TMG) as precursor by metal organic chemical vapor deposition (MOCVD). The 


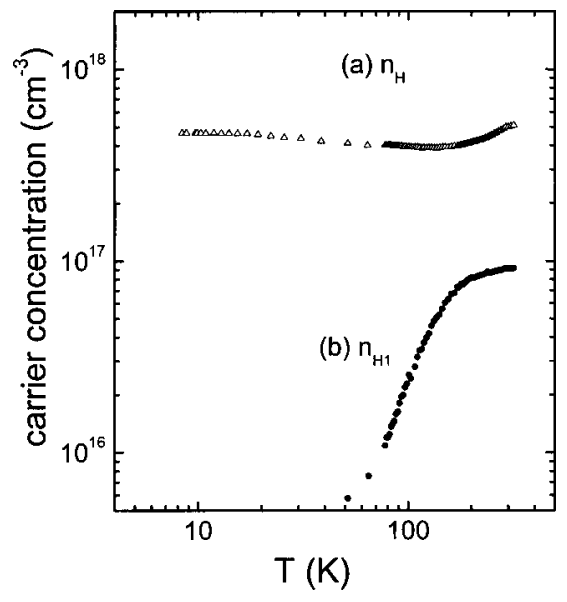

FIG. 1. Temperature dependence of (a) measured electron concentration $n_{\mathrm{H}}$. (b) Derived electron concentration $n_{\mathrm{H}}$ of GaN bulk film.

sapphire substrate was first heated to $1050{ }^{\circ} \mathrm{C}$ in a stream of hydrogen. A $\sim 300 \AA$ A thick unintentionally doped GaN buffer layer was grown at low temperature $\left(520^{\circ} \mathrm{C}\right)$. Then the system was heated to $1050^{\circ} \mathrm{C}$ and kept for $15 \mathrm{~min}$ to anneal the buffer layer. The GaN layer with the thickness of $5 \mu \mathrm{m}$ was grown at a temperature of $1050{ }^{\circ} \mathrm{C}$. The typical mole ratio of $\mathrm{NH}_{3}$ and TMG was V/III $\sim 2500$ during the deposition. More details of the growth procedure can be found in Ref. 20. The samples were cut into square van der Pauw geometry $(8 \times 8$ $\mathrm{mm}^{2}$ ). Ohmic contacts were fabricated by evaporating $800 \AA$ Al film onto the $\mathrm{GaN}$ epilayer followed by thermal annealing at $750{ }^{\circ} \mathrm{C}$ for 2 min under $\mathrm{N}_{2}$ ambient. Good ohmic contacts were verified over the whole measured temperature range. Temperature-dependent Hall effect measurements were carried out in darkness between the temperatures of 4 and $320 \mathrm{~K}$ in a magnetic field of $0.5 \mathrm{~T}$ using a BIO-RAD HL5580 Hall effect system.

Confocal micro-Raman spectroscopic measurements were carried out by the system consisting of a Spex 750M monochromator with 1800 lines $/ \mathrm{mm}$ single grating, an Olympus DX40 microscope, a Princeton Spectrum One charge-coupled device detector cooled with liquid nitrogen, and a notch filter. The Raman measurements were performed in back-scattering $Z(X,-) \bar{Z}$ geometry. The $488 \mathrm{~nm}$ line of an $\mathrm{Ar}^{+}$laser operating at a power of $200 \mathrm{~mW}$ was used for the light source. The laser beam was focused to a spot size of about $1 \mu \mathrm{m}$ with the use of a $100 \times$ objective lens and thus the spatial resolutions in the $x, y$, and $z$ directions is of the order of $\sim 1 \mu \mathrm{m}$. The instrumental spectral resolution was 1 $\mathrm{cm}^{-1}$. All the Raman spectra were taken at room temperature.

The GaN sample was also etched in $\mathrm{H}_{3} \mathrm{PO}_{4}$ at $160{ }^{\circ} \mathrm{C}$ for 15 min to study the dislocation density. The surface morphology was subsequently analyzed using a typing mode Digital Instrument III atomic force microscopy (AFM).

\section{RESULTS AND DISCUSSION}

The carrier concentration $n_{\mathrm{H}}$ and the Hall mobility $\mu_{\mathrm{H}}$ measured as a function of temperature for the present $\mathrm{GaN}$ sample are shown in Figs. 1(a) and 2(a), respectively. As

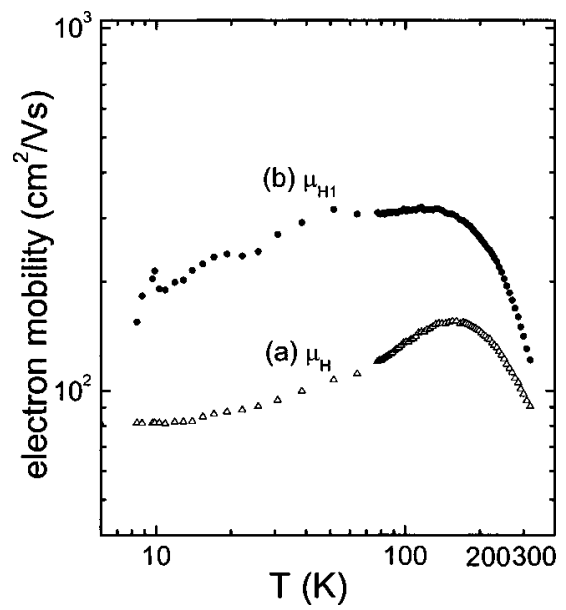

FIG. 2. Temperature dependence of (a) measured electron mobility $\mu_{\mathrm{H}}$. (b) Derived electron mobility $\mu_{\mathrm{H} 1}$ of GaN bulk film.

may be seen, the temperature-independent carrier concentration and mobility are observed at low temperature $(<20 \mathrm{~K})$. Because of parallel conduction effects, these measurements do not accurately represent the GaN epilayer properties. For two parallel conduction paths, the carrier concentration can be written as $^{21}$

$$
n_{\mathrm{H}}=\left(n_{\mathrm{H} 1} \mu_{\mathrm{H} 1}+n_{\mathrm{H} 2} \mu_{\mathrm{H} 2}\right)^{2} /\left(n_{\mathrm{H} 1} \mu_{\mathrm{H} 1}^{2}+n_{\mathrm{H} 2} \mu_{\mathrm{H} 2}^{2}\right),
$$

where, $n_{\mathrm{H} 1}$ and $\mu_{\mathrm{H} 1}$ represent the carrier concentration and mobility, respectively, in the GaN bulk film, whose contribution to conduction becomes more significant as the temperature increases, and $n_{\mathrm{H} 2}$ and $\mu_{\mathrm{H} 2}$ are associated with a low mobility, metallic channel, which dominates at low temperature. The approach to extract the parameters of each channel from Eq. (1) is to identify the metallic channel either as an impurity band, ${ }^{22}$ or as a degenerate layer at $\mathrm{GaN} /$ substrate interface, i.e., two-layer model ${ }^{10}$ in terms of the energetic and spatial distribution of mobile carriers, respectively. In the following paragraph, we find the two-layer model can well describe the TDH data and the depth dependent Raman data.

According to the Raman selection rule and for the $Z(X,-) \bar{Z}$ configuration used in the present study, $A_{1}(\mathrm{LO})$, $E_{2}$ (low), and $E_{2}$ (high) modes of hexagonal GaN are expected to be observed in the Raman spectra. However, the $E_{2}$ (low) mode is not within the covered spectral range of the present observations made. Micro-Raman spectra of the GaN film at different depth positions are shown in Fig. 3. The parameter " $D$ " is the distance between the GaN sapphire boundary and the position at which the Raman spectrum is taken. All spectra are normalized in intensity by the peak height of the $E_{2}$ (high) mode, and are shifted vertically for easy comparison. A typical Raman spectrum taken at the surface of the GaN film (i.e., $D=5 \mu \mathrm{m}$ ) is shown in Fig. 3 (a). The dominant lines are the $E_{2}$ (high) $\left(570 \mathrm{~cm}^{-1}\right)$ and $A_{1}$ (LO) $\left(735 \mathrm{~cm}^{-1}\right)$ modes. For the Raman spectrum taken at the interfacial region $D=1 \mu \mathrm{m}$ away from the GaN/ sapphire boundary as shown in Fig. 3(d), the $E_{g}\left(750 \mathrm{~cm}^{-1}\right)$ mode due to the sapphire substrate is also observed. All of 


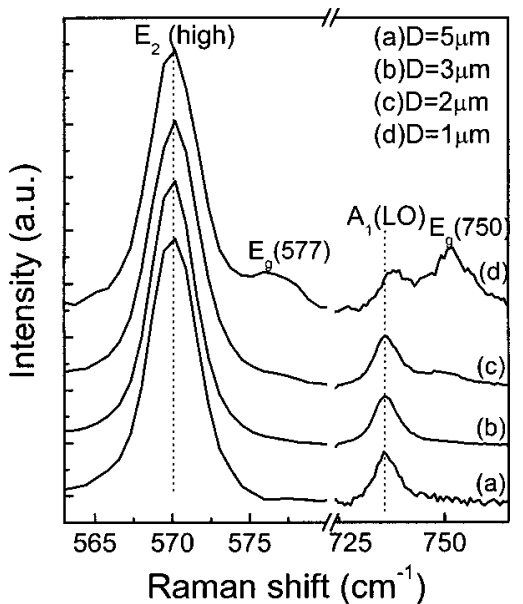

FIG. 3. Room temperature depth-profiled micro-Raman spectra of unintentionally doped GaN film grown on sapphire substrate. The parameter " $D$ " denotes the distance away from the GaN/sapphire boundary. The spectra are normalized by the peak intensity of the $E_{2}$ (high) phonon mode and shifted vertically for comparison. The dotted lines are guide to the eye.

these Raman signals observed are, in general, agreement with the previous reported Raman modes of epitaxial GaN films. ${ }^{23-27}$

It is known that the line position of the $E_{2}$ mode of $\mathrm{GaN}$ shifts significantly with the presence of $\sigma_{x x}$ stress in the film. ${ }^{28}$ It is interesting to note in Fig. 1, the peak positions of the $E_{2}$ (high) mode were found to be constant at $570 \pm 0.5$ $\mathrm{cm}^{-1}$ for all the Raman spectra taken at different depths. Using the Raman peak position of the $E_{2}$ mode of a freestanding bulk GaN sample $\left(568 \mathrm{~cm}^{-1}\right)$ (Ref. 29) and the formula $\Delta \omega=6.2 \sigma$ (where $\Delta \omega$ is the frequency shift in $\mathrm{cm}^{-1}$ units and $\sigma$ is the biaxial stress in GPa units), ${ }^{30}$ the compression stress of the present $\mathrm{GaN}$ film is found to be $0.32 \mathrm{GPa}$. The most likely explanation is that most of the strain caused by the lattice and the thermal-expansion mismatches is relaxed by the formation of dislocations close to the substratefilm interface. ${ }^{31,32}$ Complete relaxation requires misfit dislocations at an average distance of $2.0 \mathrm{~nm}$. However the dislocations formed at the GaN sapphire interface are clustered, leaving some residual strain remained in the epilayer, and resulting in some constant residual strain when the epilayer is thicker than $\sim 1 \mu \mathrm{m}$.

The intensity and peak position of the $A_{1}(\mathrm{LO})$ mode of the present GaN sample taken at the GaN surface are identical to those at the depth of $D \geqslant 2 \mu \mathrm{m}$ away from the GaN/ sapphire boundary. However, the $A_{1}(\mathrm{LO})$ mode of the spectrum taken at the $1 \mu \mathrm{m}$ interfacial region becomes more asymmetric and broadened with its peak position shifting $\left(\sim 3 \mathrm{~cm}^{-1}\right)$ to higher frequency as compared to those taken within the GaN bulk film, indicating a significant amount of coupling between the $A_{1}(\mathrm{LO})$ phonon and the heavily damped plasmon. This result is consistent with previously reported Raman studies on heavily doped n-type GaN materials, ${ }^{17,33,34}$ as well as those observed in $\mathrm{SiC}$ and $\mathrm{GaP}^{35,36}$ for which the high-frequency branch of the LO phonon-plasmon coupled mode shifts slightly to the highfrequency side with accompanying peak shape broadening as the carrier concentration increases.
It should be noted that $A_{1}(\mathrm{LO})$ peak also shifts due to stress. It was reported that a biaxial compressive stress of 1 GPa will shift the $A_{1}(\mathrm{LO})$ peak toward a higher wave number by $0.8 \mathrm{~cm}^{-1}{ }^{37}$ As was stated in the previous discussion, the GaN film in the present study has a compressive stress about $0.32 \mathrm{GPa}$ when the epilayer is thicker than $1 \mu \mathrm{m}$. This stress is expected to shift the phonon frequency of the $A_{1}$ (LO) mode upwards by $\sim 0.25 \mathrm{~cm}^{-1}$, an amount which is negligible compared to the observed $A_{1}(\mathrm{LO})$ mode shift of $\sim 3 \mathrm{~cm}^{-1}$ found at the interfacial region. The effect of stress can thus safely be disregarded if the free carrier concentration determination is estimated from the phonon-plasmon coupling mode. In this case, the carrier density $\mathrm{n}$ and the drift mobility $\mu$ can be evaluated by a line-shape fitting analysis based on the semiclassical model which involves the consideration of the deformation potential and the electro-optical mechanisms. The line shape of the LO phonon is given by $^{35,36,38}$

$$
I(\omega)=S A(\omega) \operatorname{Im}[-1 / \epsilon(\omega)],
$$

where $S$ is a proportionality factor,

$$
\begin{aligned}
A(\omega)= & 1+2 C \omega_{T}^{2}\left\lfloor\omega_{P}^{2} \gamma\left(\omega_{T}^{2}-\omega^{2}\right)-\omega^{2} \Gamma\left(\omega^{2}-\gamma^{2}-\omega_{P}^{2}\right)\right\rfloor / \\
& \Delta+\left(C^{2} \omega_{T}^{4} / \Delta\right)\left\lfloor\omega_{P}^{2}\left\{\gamma\left(\omega_{L}^{2}-\omega_{T}^{2}\right)+\Gamma\left(\omega_{P}^{2}-2 \omega^{2}\right)\right\}\right. \\
& \left.+\omega^{2} \Gamma\left(\omega^{2}+\gamma^{2}\right)\right\rfloor /\left(\omega_{L}^{2}-\omega_{T}^{2}\right),
\end{aligned}
$$

$\Delta$ being given by

$$
\begin{aligned}
\Delta= & \omega_{p}^{2} \gamma\left\lfloor\left(\omega_{T}^{2}-\omega^{2}\right)^{2}+(\omega \Gamma)^{2}\right\rfloor+\omega^{2} \Gamma\left(\omega_{L}^{2}-\omega_{T}^{2}\right) \\
& \times\left(\omega^{2}+\gamma^{2}\right),
\end{aligned}
$$

and

$$
\begin{aligned}
\epsilon(\omega)= & \epsilon_{\infty}\left\{1+\left(\omega_{L}^{2}-\omega_{T}^{2}\right) /\left(\omega_{T}^{2}-\omega^{2}-i \omega \Gamma\right)\right. \\
& \left.-\omega_{P}^{2} /\left(\omega^{2}-i \omega \gamma\right)\right\}
\end{aligned}
$$

where $\omega_{L}$ and $\omega_{T}$ are the longitudinal and transverse optical phonon frequencies, $\gamma$ is the plasmon damping constant, $\Gamma$ is the phonon damping constant, $\omega_{P}$ is the plasmon frequency, and $\mathrm{C}$ is the Faust-Henry coefficient for hexagonal GaN. ${ }^{17,39-41}$

As can be seen in Fig. 3(d), the $E_{g}$ mode due to the sapphire substrate is also observed and superimposed with the $A_{1}(\mathrm{LO})$ peak. We can remove the sapphire $E_{g}$ mode by spectral subtraction. The observed line shapes of $A_{1}(\mathrm{LO})$ coupled modes measured at the interfacial region (i.e., $D$ $=1 \mu \mathrm{m}$ ) and the bulk film (i.e., $D=5 \mu \mathrm{m}$ ) were fitted according to Eqs. (1)-(4), with $\omega_{T}, \omega_{L}$, and $C$ being fixed at the literature values of $\omega_{T}=533 \mathrm{~cm}^{-1}, \omega_{L}=735 \mathrm{~cm}^{-1}$, and $C=0.48 .^{41}$ For the case of $\mathrm{GaN}$, values of $\epsilon_{\infty}=5.35$ and $m^{*}=0.2 m_{0}$ were also taken. ${ }^{42} \Gamma, \gamma$, and $\omega_{P}$ were treated as fitting parameters. The carrier density $n$ as well as the mobility $\mu$ were then deduced from the relation $\omega_{P}$ $=\left\{4 \pi n e^{2} /\left(\epsilon_{\infty} m^{*}\right)\right\}^{1 / 2}$ and $r=(3 \pi / 8)\left(e / m^{*} \mu\right)$, respectively. The fitted curves (solid lines) and also the experimental data (broken lines) of the spectra taken at the surface and those at the interfacial region are shown in Fig. 4. Values of the fitting parameters, the derived carrier density $n$ and the derived mobility $\mu$ are shown in Table I. The electron con- 


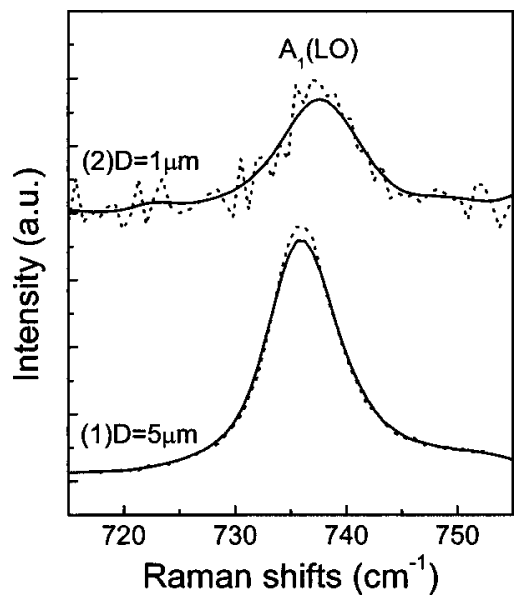

FIG. 4. Raman spectra of $A_{1}$ (LO) modes of $n$-type wurtzite GaN measured at different $D$. (1) $D=5 \mu \mathrm{m}$, (2) $D=1 \mu \mathrm{m}$. The solid lines are the modeled fitted curves of the $A_{1}$ (LO) coupled modes. The dashed lines denote experimental line shapes of the $A_{1}(\mathrm{LO})$ coupled modes.

centration and the mobility at the bulk film were found to be $n=1.5 \times 10^{17} \mathrm{~cm}^{-1}, \mu=310 \mathrm{~cm}^{2} / \mathrm{V}$ s. These results clearly confirm, in accordance with previous works, ${ }^{13,14,43}$ that there is a $1 \mu \mathrm{m}$ width highly conductive and defective interfacial region adjacent to the $\mathrm{GaN} /$ sapphire boundary having an electron concentration and the mobility of $n=8$ $\times 10^{18} \mathrm{~cm}^{-3}$ and $\mu=45 \mathrm{~cm}^{2} / \mathrm{V}$ s respectively.

The presence of the interfacial region with high carrier concentration suggests employing the two-layer model proposed by Look and Molnar ${ }^{10}$ to provide a realistic description of the present sample. In this two-layer model, layer I is taken as the GaN bulk film having a low carrier concentration and high mobility, and layer II is taken as the degenerate interfacial layer. The real electron concentration $n_{\mathrm{H}}$ and the real mobility $\mu_{\mathrm{H} 1}$ of the bulk film can be expressed as a function of the measured concentration $n_{\mathrm{H}}$, the measured mobility $\mu_{\mathrm{H}}$ the interfacial layer thickness $d$, the Hall parameters of the interfacial layer $\left(n_{\mathrm{H} 2}\right.$ and $\left.\mu_{\mathrm{H} 2}\right)$ and $n_{\mathrm{shH} 2}$ the sheet Hall concentration of interfacial layer. ${ }^{10}$

$$
\begin{gathered}
n_{\mathrm{H} 1}=\frac{\left(\mu_{\mathrm{H}} n_{\mathrm{H}}-\mu_{\mathrm{H} 2} n_{\mathrm{sh} 2} / d\right)^{2}}{\mu_{\mathrm{H}}^{2} n_{\mathrm{H}}-\mu_{\mathrm{H} 2}^{2} n_{\mathrm{sh} 2} / d}, \\
\mu_{\mathrm{H} 1}=\frac{\mu_{\mathrm{H}}^{2} n_{\mathrm{H}}-\mu_{\mathrm{H} 2}^{2} n_{\mathrm{shH} 2} / d}{\mu_{\mathrm{H}} n_{\mathrm{H}}-\mu_{\mathrm{H} 2} n_{\mathrm{shH} 2} / d} .
\end{gathered}
$$

In the calculation, the thickness $d$ of interfacial layer is taken as $1 \mu \mathrm{m}$ as revealed by micro-Raman measurement. The sheet Hall concentration $n_{\mathrm{shH} 2}$ can be measured at low enough temperature (lower than $20 \mathrm{~K}$ ) as the electrons in the bulk film freeze out and the measured Hall data are dominated by the contribution from the highly conductive interfacial layer. In this case the volume density of electrons in the interfacial layer is $n_{\mathrm{H} 2}=n_{\mathrm{shH} 2} / d=(7 \pm 0.4) \times 10^{18} \mathrm{~cm}^{-3}$. This carrier concentration coincides with those obtained from the Raman measurement described in previous paragraphs $\left(n_{\mathrm{H} 2}=8 \times 10^{18} \mathrm{~cm}^{-3}\right)$. The observed degenerate and temperature-independent characteristic of the interfacial layer [Fig. 1(a)] is also quite consistent with this carrier concentration since the Fermi level enters the conduction band when the carrier concentration is about $6 \times 10^{18} \mathrm{~cm}^{-3} .44$

The extracted concentration $n_{\mathrm{H} 1}$ and mobility $\mu_{\mathrm{H} 1}$ of the bulk film are plotted in Figs. 1(b) and 2(b), respectively. The extracted carrier concentration of the bulk film $n_{\mathrm{H} 1}$ at room temperature is $(0.9 \pm 0.4) \times 10^{17} \mathrm{~cm}^{-3}$, which agrees well with the value $1.0 \times 10^{17} \mathrm{~cm}^{-3}$ of the near surface carrier concentration obtained from the capacitance-voltage $(C-V)$ measurement on the same sample. By fitting the line shape of the $A_{1}(L O)$ Raman peak as described in the previous Raman section, the carrier concentration at the surface is 1.5 $\times 10^{17} \mathrm{~cm}^{-3}$, which also agrees well with the Hall calculated data.

In order to analyze the extracted Hall mobility of the $\mathrm{GaN}$ bulk film, the limiting effect imposed by each of the scattering mechanisms is to be considered. Assuming that the scattering events are independent of each other, the resultant mobility is given by the Matthiesen's rule: $1 / \mu=\Sigma\left(1 / \mu_{i}\right)$, where $\mu_{i}$ are the mobility contributed by the $i$ th scattering mechanism. In our first trial of fitting the mobility data $\mu_{\mathrm{H} 1}$ in Fig. 2(b), we have included ionized impurity scattering $\left(\mu_{\mathrm{ii}}\right)$, neutral impurity scattering $\left(\mu_{\mathrm{ni}}\right)$, polar optical phonon scattering $\left(\mu_{\mathrm{po}}\right)$, acoustic deformation potential scattering $\left(\mu_{\mathrm{dp}}\right)$, and piezoelectric potential scattering $\left(\mu_{\mathrm{pc}}\right)$ with the use of the well known formulas ${ }^{45,46}$ and material parameters of GaN cited in Ref. 47. It is found, however, that the fitting involving these scattering mechanisms (with $\mu_{i}$ plotted as a function of temperature in Fig. 5) cannot provide a reasonable fit to the experimental mobility data $\mu_{\mathrm{H} 1}$ of the present $\mathrm{GaN}$ bulk film. This implies other scattering mechanisms have to be considered.

Dislocation scattering in $\mathrm{GaN}$ has been studied by Weimann and Eastman ${ }^{48}$ and $\mathrm{Ng}$, Pudi, and Moustakas ${ }^{49}$ These authors showed that the mobility limited by threading dislocation scattering depends on the dislocation density and the carrier concentration. Moreover, the mobility $\mu_{\text {disl }}$ due to such scattering has a temperature dependence of $\mu_{\text {disl }} \propto T$. Dislocation scattering is thus more likely to be dominant at low temperatures giving it a similar profile to that of ionized impurity scattering. In our first mobility fitting (with only

TABLE I. Values of the fitting parameters and derived $n$ and $\mu$ for Raman $A_{1}(\mathrm{LO})$ coupled modes measured at $D=5 \mu \mathrm{m}$ and $D=1 \mu \mathrm{m}$.

\begin{tabular}{lcccccc}
\hline \hline & \multicolumn{3}{c}{ Fitting parameters } & & \multicolumn{2}{c}{ Derived $n, \mu$} \\
\cline { 2 - 3 } Raman modes & $\omega_{P}\left(\mathrm{~cm}^{-1}\right)$ & $\Gamma\left(\mathrm{cm}^{-1}\right)$ & $\gamma\left(\mathrm{cm}^{-1}\right)$ & & $n\left(\mathrm{~cm}^{-1}\right)$ & $\mu\left(\mathrm{cm}^{2} / \mathrm{Vs}\right)$ \\
\hline (1) $A_{1}(\mathrm{LO})$ measured at $D=5 \mu \mathrm{m}$ & 120 & 22 & 175 & & $1.5 \times 10^{17}$ & 310 \\
$(2) A_{1}(\mathrm{LO})$ measured at $D=1 \mu \mathrm{m}$ & 732 & 110 & 1200 & & $8 \times 10^{18}$ & 45 \\
\hline \hline
\end{tabular}




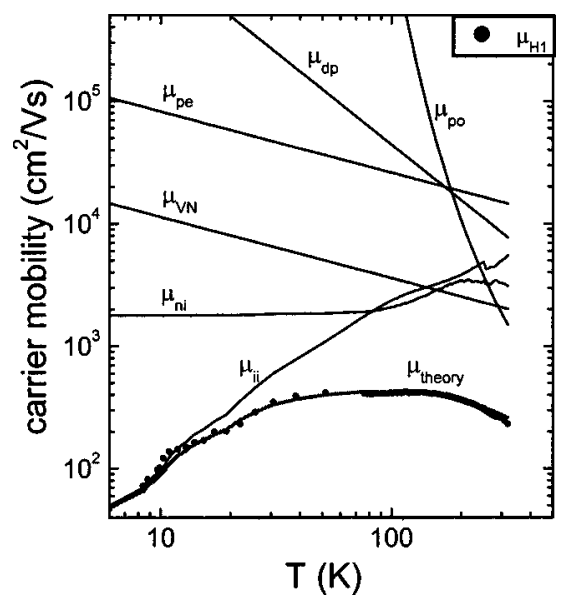

FIG. 5. The derived electron mobility of GaN bulk film $\mu_{\mathrm{H} 1}$ vs temperature, fitted in terms of various scattering mechanisms (as shown in figure). The $\mu_{\text {theory }}$ represents the theory mobility value calculated using Matthiesen's rule.

$\mu_{\mathrm{ii}}, \mu_{\mathrm{ni}}, \mu_{\mathrm{po}}, \mu_{\mathrm{dp}}$, and $\left.\mu_{\mathrm{pc}}\right)$, deviation between the modeled curve and the experimental data is found to be large at high temperature, which indicates the need for another scattering potential that would effectively limit the mobility at high temperature.

In order to investigate the dislocation density of the present GaN film, AFM images were taken after the sample was wet chemically etched with the use of $\mathrm{H}_{3} \mathrm{PO}_{4}$. Recently, Youtsey et al. ${ }^{50,51}$ demonstrated that the whisker density obtained by selectively etching GaN are very close to the effective dislocation density. Visconti et al. ${ }^{52}$ carried out a series of experiments to clarify the relation between the etch pit density and the dislocation density. They found that the effective dislocation density obtained by transmission electron microscopy was similar to the values obtained by the etching technique using hot $\mathrm{H}_{3} \mathrm{PO}_{4}$. They also pointed out, during wet etching, a careful balance must be made to ensure that every defect is delineated, and that no overetching takes place to cause merging which would lead to an underestimation of the defect density. Figure 6 shows the surface morphology of the present GaN sample observed by AFM after

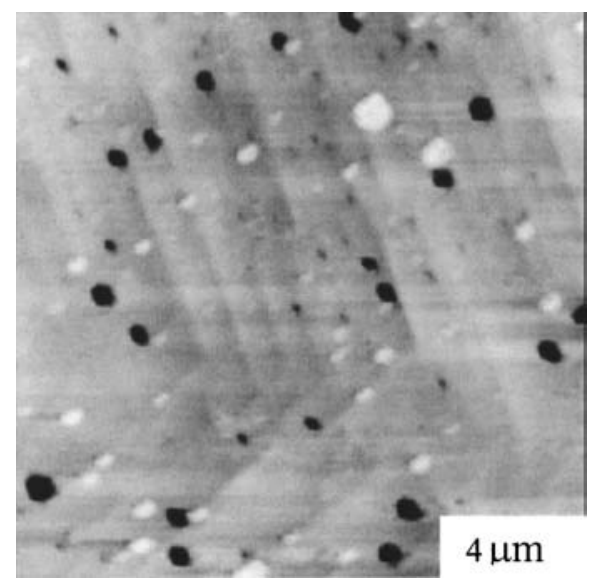

FIG. 6. $4 \times 4 \mu \mathrm{m}^{2}$ AFM image of the MOCVD grown GaN surface morphology after etching in $\mathrm{H}_{3} \mathrm{PO}_{4}$ for $15 \mathrm{~min}$ at $160^{\circ} \mathrm{C}$. the sample being etched in $\mathrm{H}_{3} \mathrm{PO}_{4}$ for 15 min at $160{ }^{\circ} \mathrm{C}$. By counting the number of etch pits per unit area, the dislocation density of the present GaN sample was found to have a value of $3-5 \times 10^{8} \mathrm{~cm}^{-2}$. This value is relatively low as compared with the dislocation density of $\sim 10^{10} \mathrm{~cm}^{-2}$ present at the poorly matched GaN sapphire interface. From the viewpoint of the two-step MOCVD growth technique which was used in fabricating the present samples, it can be concluded that the dislocations are mainly confined to the GaN/sapphire interfacial region as the growth of the low-temperature buffer layer is introduced.

The dislocation density of the present sample revealed from the AFM technique with wet etching is 3-5 $\times 10^{8} \mathrm{~cm}^{-2}$, while the scattering at charged dislocation lines only becomes significant for dislocation density above $10^{9} \mathrm{~cm}^{-2}$. This implies that dislocation scattering is not likely to be a significant scattering process in the GaN bulk film.

Zhu and Sawaki ${ }^{53}$ proposed that nitrogen vacancy $\left(V_{\mathrm{N}}\right)$ scattering should be taken into account when analyzing the electron mobility data in GaN films grown by MOCVD. Although theoretical calculation reveals that for $n$-type GaN the formation energy of $V_{\mathrm{N}}$ is large i.e., about $\left.4 \mathrm{eV}\right),{ }^{54}$ it does not exclude the possibility that the GaN materials have relatively high nitrogen vacancy concentration under certain growth conditions. Moreover, technologically it is likely that $V_{\mathrm{N}}$ is present during the growth process because of the very high nitrogen equilibrium pressure at the high growth temperature. Using a square-well potential to describe the nitrogen-vacancy-induced scattering potential, Zhu and Sawaki ${ }^{53}$ showed that the mobility $\left(\mu_{V_{N}}\right)$ caused by this scattering follows $\mu_{V_{N}} \propto T^{-1 / 2}$ and thus this kind of scattering has the derived form of being dominant at high temperatures. In addition, Chua et al. ${ }^{55}$ reported that $V_{\mathrm{N}}$ were created in stoichiometric GaN film by plasma exposure and also showed the mobility limited by nitrogen vacancy scattering follows $\sim T^{-1 / 3}$, which is close to the result predicted by Zhu and Sawaki ${ }^{53}$

In the final mobility fitting (shown in Fig. 5), $V_{\mathrm{N}}$ scattering was taken into account by including the $V_{\mathrm{N}}$ limiting mobility $^{53}$

$$
\mu_{V_{\mathrm{N}}}=\frac{9}{16 \sqrt{3}} \frac{h^{4} e}{m^{* 5 / 2} N_{V_{\mathrm{N}}}} \frac{1}{U_{0}^{2} a^{6}}\left(k_{B} T\right)^{-1 / 2} .
$$

The compensated acceptor concentration $\left(N_{\mathrm{A}}\right)$, and the nitrogen vacancy concentration $\left(N_{V_{N}}\right)$ are treated as the free parameters in the fitting process. A least squares fit of the mobility data yielded a good agreement over the entire temperature range with fitted values of $N_{\mathrm{A}}=(5.8 \pm 0.5)$ $\times 10^{16} \mathrm{~cm}^{-3}$ and $N_{V_{\mathrm{N}}}=(1.7 \pm 0.5) \times 10^{17} \mathrm{~cm}^{-3}$. The results indicate that the concentration of nitrogen vacancies is relatively high in the bulk film of the present GaN sample and nitrogen vacancies play a significant role in limiting the electron mobility.

With the charge neutrality equation, the donor concentration $N_{D}$ and the donor ionization energy $E_{D}$ are given by 


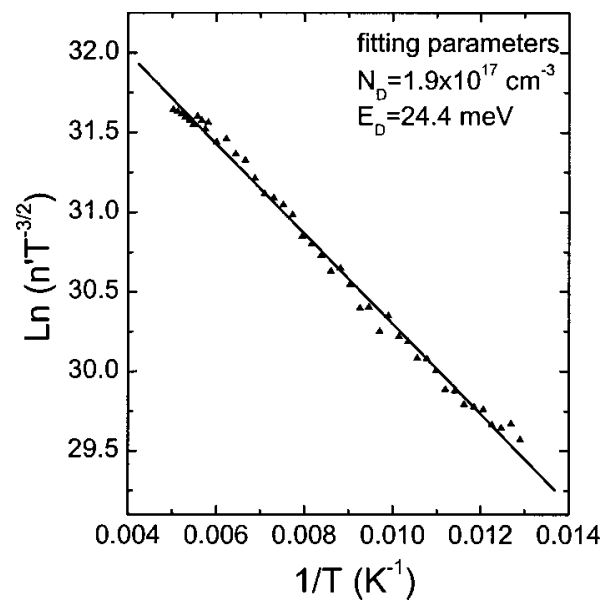

FIG. 7. Linear fitting of charge neutrality equation, in which $N_{D}$ and $E_{D}$ are fitting parameters, $N_{\mathrm{A}}=5.8 \times 10^{16} \mathrm{~cm}^{-3}$ is independently obtained by mobility fitting.

$$
\begin{aligned}
\frac{n_{\mathrm{H} 1}\left(n_{\mathrm{H} 1}+N_{\mathrm{A}}\right)}{N_{D}-n_{\mathrm{H} 1}-N_{\mathrm{A}}}=n^{\prime} & =\frac{N_{c}}{\beta} \exp \left(-\frac{E_{\mathrm{D}}}{K T}\right) \\
& =\frac{1}{\beta} N_{C}^{0} T^{3 / 2} \exp \left(-\frac{E_{\mathrm{D}}}{K T}\right) .
\end{aligned}
$$

Plot of $\left(n^{\prime} T^{3 / 2}\right)$ as a function of $1 / T$ is shown in Fig. 7 with the values of $n^{\prime}$ calculated from the fitted parameters. Since $N_{\text {A }}$ was independently determined by the mobility fitting process, the reliability and the accuracy of the fitting values of $N_{D}$ and $E_{D}$ can be improved by the reduction in the number of fitting parameters. The donor concentration and the donor activation energy were found to be $N_{\mathrm{D}}=(1.9 \pm 0.2)$ $\times 10^{1} \mathrm{~cm}^{-3}$ and $E_{D}=24 \mathrm{meV}$, respectively. With regard to $E_{D}$, the donor potential can be screened by free and bound charges, and the binding energy in the unscreened dilute limit $E_{D 0}$ is given by $E_{D 0}=E_{D}+\alpha N_{D}^{1 / 3}$, where $\alpha$ is an empirically determined screening parameter dependent on the semiconductor. Since for $\mathrm{GaN} \alpha \approx 2.1 \times 10^{-5} \mathrm{meV} \mathrm{cm},{ }^{56}$ the unscreened donor binding energy can be calculated as $E_{D 0}$ $=32 \mathrm{meV}$. This value is close to the values of $\mathrm{Si}$ or $\mathrm{O}$ shallow donors reported in the literature. ${ }^{16,57,58}$ This implies $\mathrm{O}$ or $\mathrm{Si}$ as possible residual donors in the present sample.

As the fitting described in previous paragraphs gives the concentration of compensating acceptors $N_{\mathrm{A}}=5.8$ $\times 10^{16} \mathrm{~cm}^{-3}$ in the present sample, the acceptor to donor ratio is $N_{\mathrm{A}} / N_{D}=0.31$. Considering the mobility limiting mechanisms of ionized impurity scattering, piezoelectric scattering, acoustic phonon, and polar optical phonon scattering, Chin, Tansley, and Osotchan ${ }^{59}$ have calculated the carrier mobility of $\mathrm{GaN}$ as a function of temperature for different $N_{\mathrm{A}} / N_{D}$ ratio. It is noticed that with the present $N_{\mathrm{A}} / N_{D}$ ratio, the calculated mobility given by Chin, Tansley, and Osotchan ${ }^{59}$ is higher than the experimental data obtained in the present study. This implies the observed low carrier mobility in the present study is not only due to the heavy compensation but is also a consequence of other scattering processes, such as dislocation, stacking fault scattering (in the interfacial region) and nitrogen vacancy scattering (in the bulk film).

\section{CONCLUSION}

In conclusion, we have studied the unintentionally doped GaN films grown on sapphire with the use of the depth profiled micro-Raman spectroscopy, the AFM technique combined with wet etching, and temperature-dependent Hall measurements. The decrease of the peak intensity and the asymmetrical broadening of $A_{1}(\mathrm{LO})$ phonon line taken at the depth close to the near GaN/sapphire interface region ( $D$ $\leqslant 1 \mu \mathrm{m}$ ) indicates the presence of a highly conductive interfacial layer. The carrier concentrations of the bulk film and also the interfacial layer were determined by fitting the line shape of the $A_{1}(\mathrm{LO})$ coupled modes. Based on the presence of this conductive interfacial layer, a two-layer model has been used to analyze the temperature-dependent Hall data. The carrier concentration and the mobility of the GaN/ sapphire interfacial region and GaN bulk film determined by the Raman method are found to agree well with those obtained from Hall data analysis. Detailed mobility analysis strongly suggests that nitrogen vacancy scattering has an important electron mobility limiting effect in the $\mathrm{GaN}$ bulk film. The Hall data also confirm that dislocation scattering is dominant in the interfacial region, but is not the important scattering mechanism in the GaN bulk film, which coincides with the low dislocation densities found in the GaN bulk film revealed by the chemical etching method.

\section{ACKNOWLEDGMENTS}

The work described in this paper is partially supported by the grant from the Research Grant Council of the Hong Kong Special Administration Region, China (under project Nos. 7085/01P, HKU1/00C, and HKU7103/02P) and the Hung Hing Ying Physical Science research found.

${ }^{1}$ U. V. Bhapkar and M. S. Shur, J. Appl. Phys. 82, 1649 (1997).

${ }^{2}$ H. Sakai et al., Jpn. J. Appl. Phys., Part 2 34, L1429 (1995).

${ }^{3}$ L. S. McCarthy, P. Kozodoy, M. J. W. Rodwell, S. P. DenBaars, and U. K. Mishra, IEEE Electron Device Lett. 35, 277 (1999).

${ }^{4}$ E. M. Chumbes, J. A. Smart, T. Prunty, and J. R. Shealy, IEEE Trans. Electron Devices 48, 416 (2001).

${ }^{5}$ M. A. Khan, M. Shur, J. N. Kuznia, Q. Chen, J. Burm, and W. Schaff, Appl. Phys. Lett. 66, 1083 (1995).

${ }^{6}$ B. E. Foutz, L. F. Eastman, U. V. Bhapkar, and M. S. Shur, Appl. Phys. Lett. 70, 2849 (1997).

${ }^{7}$ H. Amano, N. Sawaki, I. Akasaki, and Y. Toyada, Appl. Phys. Lett. 48, 353 (1986).

${ }^{8}$ H. Amano, J. Akasaki, K. Hiramatsu, and N. Koide, Thin Solid Films 163, 415 (1988).

${ }^{9}$ S. Nakamura, Jpn. J. Appl. Phys., Part 1 30, L1705 (1991).

${ }^{10}$ D. C. Look and R. J. Molnar, Appl. Phys. Lett. 70, 3377 (1997).

${ }^{11}$ J. W. P. Hsu, D. V. Lang, S. Richter, R. N. Kleiman, A. M. Sergent, and R. J. Monlar, Appl. Phys. Lett. 77, 2873 (2000).

${ }^{12}$ C. Mavroidis, J. J. Harris, M. J. Kappers, N. Sharma, C. J. Humphreys, and E. J. Thrush, Appl. Phys. Lett. 79, 1121 (2001).

${ }^{13}$ M. Ilegems and H. C. Montgomery, J. Phys. Chem. Solids 34, 885 (1973).

${ }^{14}$ C. Mavroidis, J. J. Harris, M. J. Kappers, C. J. Humphreys, and Z. Bougrioua, J. Appl. Phys. 93, 9095 (2003).

${ }^{15}$ S. H. Goss, X. L. Sun, A. P. Young, L. J. Brillson, D. C. Look, and R. J. Molnar, Appl. Phys. Lett. 78, 3630 (2001).

${ }^{16}$ X. L. Xu et al., Appl. Phys. Lett. 76, 152 (2000).

${ }^{17}$ T. Kozawa, T. Kachi, H. Kano, Y. Taga, M. Hashimoto, N. Koide, and K. Manabe, J. Appl. Phys. 75, 1098 (1994).

${ }^{18}$ M. V. Klein, B. N. Ganguly, and P. J. Colwell, Phys. Rev. B 6, 2380 (1972). 
${ }^{19}$ F. A. Ponce, J. W. Steeds, C. D. Dyer, and G. D. Pitt, Appl. Phys. Lett. 69, 2650 (1996).

${ }^{20}$ Y. Huang, X. D. Chen, S. Fung, C. D. Beling, and C. C. Ling, J. Appl. Phys. 94, 5771 (2003)

${ }^{21}$ R. L. Petritz, Phys. Rev. 110, 1254 (1958).

${ }^{22}$ R. Molnar, T. Lei, and T. D. Moustakas, Appl. Phys. Lett. 62, 72 (1993).

${ }^{23}$ Z. C. Feng, M. Schurman, and R. A. Stall, J. Vac. Sci. Technol. A 15, 2428 (1997).

${ }^{24}$ F. A. Ponce, D. P. Bour, W. Gotz, and P. J. Wright, Appl. Phys. Lett. 68, 57 (1996).

${ }^{25}$ M. S. Liu, L. A. Bursill, S. Prawer, K. W. Nugent, Y. Z. Tong, and G. Y. Zhang, Appl. Phys. Lett. 74, 3125 (1999).

${ }^{26}$ T. Azuhata, T. Sota, K. Suzuki, and S. Nakamura, J. Phys. Chem. 7, L129 (1995).

${ }^{27}$ D. Behr, J. Wagner, J. Schneider, H. Amano, and I. Akasaki, Appl. Phys. Lett. 68, 2404 (1996).

${ }^{28} \mathrm{H}$. Siegle, Gitterdynamik und Defekte in Gallium- und Alumiumnitrid (Wissenschaft und Technik, Berlin, 1998).

${ }^{29}$ P. Perlin, C. J. Carillon, J. P. Itie, A. S. Miguel, I. Grzegory, and A. Polian, Phys. Rev. B 45, 83 (1992).

${ }^{30}$ T. Kozawa, T. Kachi, H. Kano, H. Nagase, N. Koide, and K. Manabe, J. Appl. Phys. 77, 4389 (1995).

${ }^{31}$ S. C. Jain, A. H. Harker, and R. A. Cowley, Philos. Mag. A 75, 1461 (1997).

${ }^{32}$ S. C. Jain, K. Pinardi, H. E. Maes, R. Van Overstraeten, M. Willander, and A. Atkinson, Mater. Res. Soc. Symp. Proc. 482, 875 (1998).

${ }^{33}$ M. Pophristic, F. H. Long, M. Schurman, J. Ramer, and I. T. Ferguson, Appl. Phys. Lett. 74, 3519 (1999).

${ }^{34}$ D. Kirillov, H. Lee, and J. S. Harris, Jr., J. Appl. Phys. 80, 4058 (1996).

${ }^{35}$ G. Irmer, V. V. Toporov, B. H. Bairamov, and J. Monecke, Phys. Status Solidi B 119, 595 (1983).

${ }^{36}$ M. V. Klein, B. N. Ganguly, and P. J. Colwell, Phys. Rev. B 6, 2380 (1972).

${ }^{37}$ F. Demangeot, J. Frandon, M. A. Renucci, O. Briot, B. Gil, and R. L. Aulombard, Solid State Commun. 100, 207 (1996).

${ }^{38}$ D. T. Hon and W. L. Faust, Appl. Phys. 1, 241 (1973).
${ }^{39}$ W. L. Faust and C. H. Henry, Phys. Rev. Lett. 17, 1265 (1996).

${ }^{40}$ F. Demangeot, J. Frandon, M. A. Renucci, N. Grandjean, B. B. Beaumont, J. Massies, and P. Gibart, Solid State Commun. 106, 491 (1998).

${ }^{41}$ H. Harima, H. Sakashita, and S. Nakashima, Mater. Sci. Forum 264-8, 1363 (1998).

${ }^{42}$ A. S. Barker, Jr. and M. Ilegems, Phys. Rev. B 7, 743 (1973).

${ }^{43}$ C. Mavroidis, J. J. Harris, R. B. Jackman, I. Harrison, B. J. Ansell, Z. Bougrioua, and I. Moerman, J. Appl. Phys. 91, 9835 (2002).

${ }^{44}$ T. Matsubara and Y. Toyozawa, Prog. Theor. Phys. 26, 739 (1961).

${ }^{45}$ D. C. Look, Electrical Characterization of GaAs Materials and Devices (Wiley, New York, 1989).

${ }^{46}$ D. L. Rode, Low Field Electron Transport, Semiconductor and Semimetals Vol. 10 (Academic, New York, 1973).

${ }^{47}$ D. K. Gaskill, L. B. Rowland, and K. Doverspike, in Properties of Group III Nitrides EMIS series No. 11, edited by James H. Edigar (INSPEC, London, 1994), p. 101.

${ }^{48}$ N. G. Weimann et al., J. Appl. Phys. 83, 3656 (1998).

${ }^{49}$ H. M. Ng et al., Appl. Phys. Lett. 73, 821 (1998).

${ }^{50}$ C. Youtsey, L. T. Romano, and I. Adesida, Appl. Phys. Lett. 73, 797 (1998).

${ }^{51}$ C. Youtsey, L. T. Romano, R. J. Molnar, and I. Adesida, Appl. Phys. Lett. 74, 3537 (1999).

${ }^{52}$ P. Visconti et al., Mater. Sci. Eng., B 93, 229 (2002).

${ }^{53}$ Q.-S. Zhu and N. Sawaki, Appl. Phys. Lett. 76, 1594 (2000).

${ }^{54}$ J. Neugebauer and C. G. Van de Walle, Phys. Rev. B 50, 8067 (1994).

${ }^{55}$ S. J. Chua, H. W. Choi, J. Zhang, and P. Li, Phys. Rev. B 64, 205302 (2001).

${ }^{56}$ B. K. Meyer, D. Volm, A. Graber, H. C. Alt, T. Detchprohm, A. Amano, and I. Akasaki, Solid State Commun. 95, 597 (1995).

${ }^{57}$ Gallium Nitride (GaN) I. Semiconductors and Semimetals Vol. 50, edited by J.I. Pankove and T. Moustakas (Academic, San Diego, 1998), p. 283.

${ }^{58}$ W. Götz, R. S. Kern, C. H. Chen, H. Liu, D. A. Steigerwald, and R. M. Fletcher, Mater. Sci. Eng., B 59, 211 (1999).

${ }^{59}$ V. W. L. Chin, T. L. Tansley, and T. Osotchan, J. Appl. Phys. 75, 7365 (1994). 